\title{
A computer-graphic technique for the study of body size perception and body types
}

\author{
PHILIP J. BENSON \\ University Laboratory of Physiology, Oxford, England \\ JOANNE L. EMERY \\ University of Newcastle, Newcastle-upon-Tyne, England \\ ESTHER M. COHEN-TOVÉE \\ St. George's Hospital, Morpeth, England \\ and \\ MARTIN J. TOVÉE \\ University of Newcastle, Newcastle-upon-Tyne, England
}

\begin{abstract}
We present a novel approach for measuring body size estimation in normal and eating-disordered women and men. Clinical categories of body types were used as prototypes. By comparing the subjective appearance of a person's body with prototypes, we can understand how different attributes of his or her body shape contribute to perception of body size. After lifelike random distortions have been applied to parts of their body image, individuals adjust their body shapes until they converge on their perceived veridical appearance. Exaggeration and minimization of particular body areas measured with respect to their true shape and with different prototypes can be expressed as numerical deviations. In this way, perceived body size and body attractiveness can be appraised during the course of diagnosis and treatment of eating disorders.
\end{abstract}

External physique has a profound effect on us, influencing our perceptions of ourselves and the attitudes of others toward us. Since body morphology is related to a number of personality and social factors (Janssen \& Whiting, 1984; Kellet, Marzillier \& Lambert, 1981; Tucker, 1984), the psychosocial impact of belonging to a distinct group ( $\mathrm{so}$ matotype) can have a profound effect on individuals.

In contrast to our actual physical appearance, perceived somatotype is a component of our body image. A perceptual distortion or disturbance in body image signifies a discrepancy between how the body is imagined to look and its actual physical dimensions. When conflicting representations exist, clinical intervention may be necessary, to avoid potentially chronic effects of adapting to attributed somatotypes - for example, starvation or bingeing, anorexia and bulimia nervosa, and overeating and obesity.

A reliable and accurate method is required for the assessment of self-image and image of others in relation to

This work was supported in part by the U.K. Medical Research Council (MRC) and the Oxford MRC and McDonnell-Pew Centre for Cognitive Neuroscience. J.L.E. was supported by a Newcastle University research grant. We also thank the Charles Strasser Foundation for an equipment grant. Correspondence concerning this article should be addressed to P. J. Benson, University Laboratory of Physiology, Parks Road, Oxford, OX1 3PT, England (e-mail: philip.benson@physiol.ox.ac,uk). Further details regarding software availability and distribution costs are available from http://www.physiol.ox.ac.uk/ pjb. the self. The affective (emotional) and attitudinal components of body image are measured, using factor and cluster analysis to determine the boundaries between perceived somatotypes. Subjective ratings are frequently used to quantify differences between normal and clinical attitudes.

An alternative is to assess body size directly. Behavioral tasks in body size estimation deal with the perceptual component of body image. This is done by operationalizing body image distortion as inaccuracies in the judgment of personal body dimensions. Body part methods yield separate size estimates of different body regions, such as width of the chest, waist, hips, and thighs. Comparisons with actual sizes yield accuracy scores. Part methods have included making measurements with large callipers (Reitman \& Cleveland, 1964; Slade \& Russell, 1973), marking images (Askevold, 1975), and estimating part widths with light beams (Thompson \& Spana, 1988). Since these methods involve having the experimenter or the assistant conduct measurement making, they are not particularly appropriate. We actually wish to quantify self-perception. The methods above may be further open to question if operator training is required, because this might invalidate the technique for use in self-assessment where users are inexperienced.

The most significant drawback with parts methodology is that estimates are made in isolation from the context of a whole body and the observer is provided with no direct qualitative feedback. Conversely, whole body or 
holistic methods tend only to yield one accuracy score (or two, if profile views are estimated)-an under- or overestimation of veridicality (thinner or fatter). To date, computer methods are rather limited, and approaches have, in general, dealt with optical distortions of the body surface. These have included distorting mirrors (Traub \& Orbach, 1964) and anamorphic lenses (Glucksman \& Hirsch, 1969) or video image distortion (Freeman, Thomas, Solyom, \& Hunter, 1984). Without additional measurement, distortion methods cannot measure body parts. Distortion is also unrealistic and limited in range and direction (thinner, fatter), owing to misalignment between body parts and other clues to deformation that may be present (Taylor, Baldwin, \& Cooper, 1985).

Recent attempts to improve computer-based techniques have involved body part and whole body techniques. This is a positive step, since the clinical presentations of patients reveal that their disposition is often related to particular aspects of body image - cognitive and physical. Body Build (Dickson-Parnell, Jones, Braddy, \& Parnell, 1987) provides an outline of a profile and frontal image of a male or female body, the individual parts of which can be made thinner or fatter by altering the arc size used to draw that part. The Body Image Testing System (Schlundt \& Bell, 1993) works on a similar principle by generating body silhouettes via a series of overlapping ellipsoids. Both methods produce disappointing and unrealistic results, if only for the simple fact that a real, recognizable body image is not presented. Without the facility to alter a real body image, these procedures cannot provide a measure of actual body size with which it may be compared.

We propose a new approach, based on shape transformation and image warping, that has already been used to examine facial information (Benson, 1992, 1994a, 1994b; Benson \& Perrett, 1991a, 1991b, 1993). In doing so, we address the shortcomings of other approaches and can easily specify the goals that should be met: (1) A real image of the body should be presented; (2) interaction with that image should be realistic; (3) individual body parts should be identifiable and modifiable; (4) the system should have a simple, intuitive, and meaningful look and feel when used; (5) measurement accuracy scores obtained should be effective in contributing to descriptive statistics; (6) experimental data should yield evidence that the methodology can provide meaningful interpretations of perception of body shape and that the data are replicable; and (7) the system should be consistent in its treatment of male and female bodies.

\section{METHOD}

We first describe how body shape is altered by referring to prototypes. A procedure based on facial image caricaturing was used (Benson \& Perrett, 1991b), in which an individual's face was compared with a stan- dardized prototype and differences between features were noted. These differences were exaggerated or minimized and applied to the original face. Body shape can be altered in the same way. Instead of caricaturing the body, our aim is to study alteration of body shape according to different prototypes, while retaining the appropriate relationships between body parts.

\section{Defining Body Categories and Reference Prototypes}

Our database consists of $200+$ female and $120+$ male body images. New images are added during the normal course of our research project. We also maintain a database of body weight and height information for each individual. Data are stored anonymously. Together, this information is used to categorize body types.

We use the Body Mass Index (BMI) to categorize male and female bodies. Use of the BMI is appropriate, since its use is widespread and will be meaningful to investigators in the field. BMI is an objective measure of body weight and mass obtained by dividing weight $(\mathrm{kg})$ by height squared $(\mathrm{m})$. In women, $<19$ is classified as underweight, $20-25$ is normal, and $>25$ is classified as overweight. Scales for males are obtained in a similar way. Although BMI is a continuous measure, it is feasible to use discrete values within the range to derive specific categories of body type. Below, we will describe how this can be refined.

\section{Obtaining Body Images}

The subjects were dressed in close-fitting, but not tight, pale-colored, matte leotards and leggings. A range of clean, variously sized leotards and leggings are provided as a matter of course, to provide the most comfortable and distortion-free fit. Elastic waistbands on leggings were removed.

High-quality full-figure images are captured with a SuperVHS video camera. Three poses are assumed during filming. First, a front view, with the arms raised by approximately $50^{\circ}$, palms to camera. Legs are parted by a comfortable distance (approximately $0.5 \mathrm{~m}$ ). Second, a side view, with the arms down the side of the body. The arms are kept within the outline of the body (torso, buttocks, and thighs) and with the palms facing inward. Third, a side tarms view is filmed. This is a side view, but with the arms raised into the horizontal plane, palms facing downward. The front and side views are similar to those struck by individuals when they are in front of a mirror.

A uniform matte black drape is arranged as a side and background; the drape extends onto the floor area where the subject stands. Standing position is marked on the floor with drawing pin heads. Video camera and tripod are at a fixed distance of $3.6 \mathrm{~m}$ from the subjects' frontoparallel plane and at a torso height of $1.1 \mathrm{~m}$. No appreciable lens distortion of the figure is present. Uniform ceiling and floor lighting is used. 
Single images of each body view are captured from live video input or from tape playback, in 24-bit (16.7 million) color at $768 \times 576$ pixel resolution on a Silicon Graphics Indy workstation (100-MHz MIPS R4000 processor, Vino video option).

\section{Image Calibration}

Test images of a white card placed in the subjects' location are taken to determine camera white balance, and gamma correction of the body images is made, as necessary. Our images also contain orthogonal calibration bars. Fifty $\mathrm{cm}$ strips of white tape are affixed to the walls, ceiling, and floor. The length of these bars, in pixels, can be calculated and used as a factor in calculating body dimensions in the image plane. When the body is presented in the interface during an experiment, software automatically crops the image, to exclude these bars.

\section{Identification of Body Parts}

The body is located in the image by delineation around its features and boundary (Benson \& Perrett, $1991 \mathrm{~b}$ ). The operator is guided by a reference template while delineating each view. Automated methods for feature extraction were considered inappropriate, owing to the varieties of human figures and the need to use fixed feature sets. Manual delineation produces excellent results and takes about 5-8 min per view, depending on experience. Each view is described by a predetermined number of points chosen to capture the subtle geometry of each feature (Figure 1).

We identified individual interconnected body parts. Each is delineated in turn. Every individual's body part is described by the same set of points for each of the views, but different parts requires different numbers of points, to ensure smooth and accurate delineation.

Front and side view features are slightly different. This has come about because of two considerations. First, features were selected on the basis of verbal labels that are commonly given to body parts and that are referred to in discussions of body appearance. A survey of all the body categories allowed a uniform set of features to be identified, so that all the body images could be treated in the same way. This first consideration was the most important. Not all features can be identified in the same way in front and side views; hence, some differences are present.

Second, body features may be open to analytical interpretation in the future. This approach to body part identification depends on the utility of the present specification. Clinical data showing a relationship between perception of body image and distortions applied to body parts might lead to a better understanding of the role of body parts. One could, for example, look for consistent discontinuities in body outline and then infer definition of parts and features in terms of convexities and concavities between areas of the body outline.

In the same way that a prototypical face can be calculated by averaging matching points, average bodies and


Figure 1. Figure delineation: Body images are delineated manually. Outline figures are produced by joining the points in order. Three views of each body are captured. The left figure shows the front view (131 points), with the side +arms view shown on the right ( 83 points). A side body view is also captured ( 79 points), in which the subjects have their arms by their sides. Individual body parts are illustrated by alternate thick and thin lines. Figures such as those shown here are presented as references (templates) during the delineation procedure, with each control point in a different color to denote point ordering. In this way, every body part is entered into the database in a predetermined order.

parts are calculated for each view within each BMI category. Although the images were captured in a standardized way, simply averaging appropriate points ignores slight differences in orientation between individual's poses. We therefore normalize each feature before averaging by reorienting and scaling along its principal axis (see Figure 2). An arbitrary choice to normalize in the horizontal plane is used.

\section{Body Shape Manipulation}

The purpose of the system is to understand the association between estimations of the true (veridical) appearance of body shape and mental representations of body image. Therefore, if the user makes an overestimation of veridical shape, our aim is to simulate an increase in body mass; underestimation of veridicality requires representation of a decrease in mass. We simulate changes in mass by altering feature shape in the image plane.

The body parts in each prototype are used as references to change the shape of body parts in the veridical image. Since the whole body image is displayed, the user obtains an overall impression of how each body part relates to the whole. Altering body appearances requires a two-stage process: calculation of shape change in response to user interaction with that feature and image mapping of that request for a shape change.

Interaction with body parts is performed with graphical sliders on the user interface. Sliders embrace positive and negative dimensions, as well as magnitude. Movement of a slider changes the shape of its associated feature by specifying a value that is an over- (positive) or 


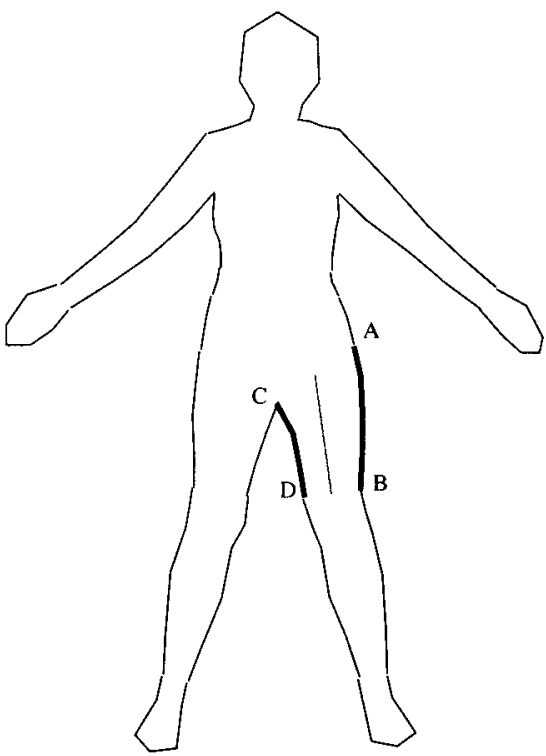

A

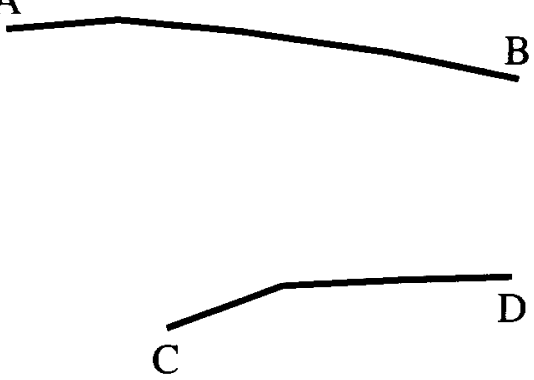

Figure 2. Normalization of body parts: Comparison between body parts of different figures is achieved by first standardizing the features. A thigh feature is shown before and after rotation of the feature midline about its center of moment in the horizontal plane. The scale of the feature being compared with a prototype is arbitrary, as is rotation, since it is rescaled to fit the original figure. By repeating the normalization step for many figures, it is possible to calculate the average dimensions of each body part and thus derive prototypical features. All the body parts are treated in the same way.

an under- (negative) estimation of veridicality (zero). A user will manipulate his or her body appearance with respect to concepts of the self and of the somatotypes. The procedure, therefore, is designed to reflect these beliefs, by comparing the veridical figure with the prototypes.

There are two approaches to changing the shape of body parts. Both are available in our system. This provision allows slightly different questions to be asked by the experimenter that address local (body part) and global (body type) issues. The first option is local and computes changes to the veridical body with respect to its BMI category prototype. Perceptual deviations from the appropriate prototype lend themselves to interpretations delineated by a single reference point suitable for that particular individual. The second option is to alter body shape in a global sense, by referring to all prototypes simultaneously. Changes in the domain of all reference body types are likely to embrace somatotypes in a more general sense.

Single prototypes. Using the local model, changes to body parts are constrained by the proximity of the target body to the prototype for the individual's BMI category. Small differences can only be enhanced or diminished by small amounts, compared with substantial changes made to larger differences that might reflect attitudinal sensitivities of the subject to those features and a desire to normalize or minimize physical appearance.

Calculating alterations to body part sizes is trivial. Each slider value is a scaling factor applied to the difference between pairs of points describing the veridical feature and the prototypical feature. A positive value increases the difference and increases the size of the feature, whereas a negative value increases the size difference in the opposite direction, tending toward no size

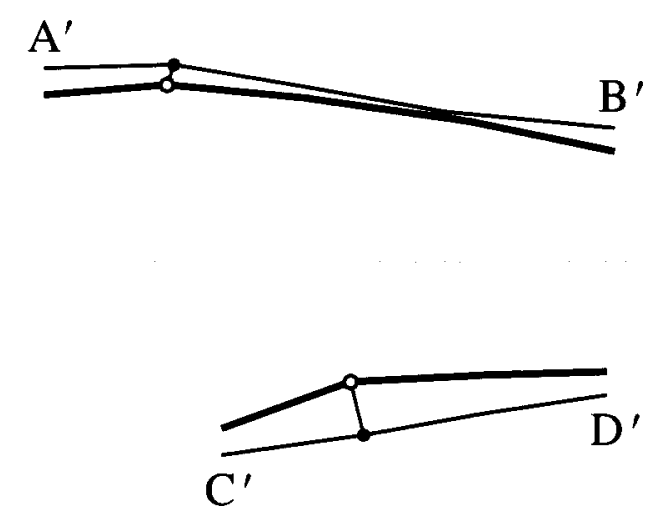

Figure 3. Calculation of shape change with a single prototype: Thick lines and open circles represent the prototype feature (Figure 2). Thin lines and filled circles are from a veridical feature. $A$ pair of sample points is shown on each side of the feature. After normalization, differences between pairs of feature points are exaggerated or diminished according to the value of the associated feature slider in the user interface. In this example, exaggeration will increase the size difference between the veridical and the prototype points along the line (vector) joining the feature points, pushing it further away from the prototype. After shape change is calculated, each body part is rendered in the image. The single prototype method examines body perception within localized features. 
difference - that is, $-100 \%$ size difference. Figure 3 illustrates this for a front-view thigh feature. As is the case when calculating prototypical features, computation is performed on normalized coordinates before being applied to the veridical data.

Multiple prototypes. A more complex procedure is required for multiple prototypes in the global model. User changes to a feature slider influence the morphology of the body part between prototypes, as well as its size and shape (Figure 4). Some preprocessing is required to establish the paths between prototypes. Interpolation is performed with splines, so that a feature's shape path passes through the feature points of each prototype. Extrapolation beyond the emaciated and obese prototypes is also calculated, to provide an estimate of body shape beyond the extreme categories.

The starting points for the veridical figure in the body shape continuum is determined by calculating the closest (statistical least-squares) fit for each veridical body part with its prototype path. In this way, individual features follow paths starting at different positions, and every change is made relative to their veridical appearance. The slider value creates a proportional deviation from the veridical position. A positive slider change will cause an increase in the size of a feature that is bigger than the individual's prototypical feature, and vice versa.
Shape changes are generated by translating the body part along the interpolated path between prototypes.

The benefit of using multiple prototypes is that changes in body shape are made in a manner that is not necessarily linear-but nevertheless increasing or decreasing - and that follows the path of shape changes that evolve between BMI prototypes. An immediate limitation is that it takes time to establish a database large enough to extract stable prototypes with minimal variability within each and with similarity between categories. Our databases have already achieved this.

\section{Image Rendering}

Imaging shape changes are performed by digital image warping adapted to the subtleties of geometric body shapes (Benson 1992; Benson \& Perrett, 1991b; cf. Wolberg, 1990). Feature points delineated on the veridical image delimit a specific area in the source imagethe body part concerned. The distorted image feature is determined by the position of feature landmarks calculated by using the non-veridical value of the respective feature slider. We change the shape and texture of a body part by warping the veridical portion of the image into the distorted shape.

An optimized implementation of the Delaunay triangulation is used to tessellate the feature points into a two-

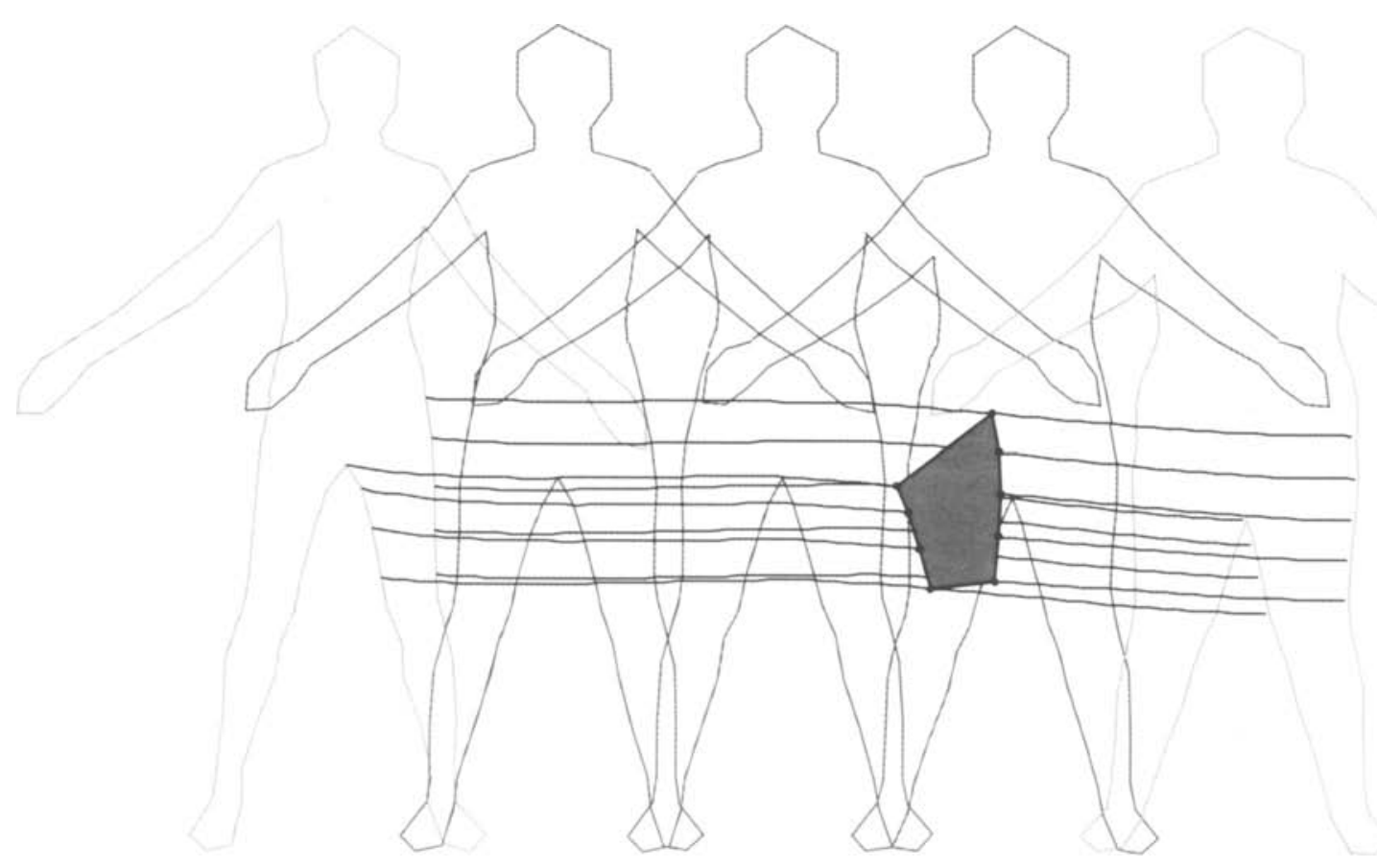

Figure 4. Calculation of shape change using multiple prototypes: Shape changes between different body size prototypes are sensitive to global changes in body type category. Prototypes from left to right show BMI $<19$ (emaciated) through BMI $>25$ (obese). Spline interpolation between each body part defines a path in which feature shape changes occur. Extrapolation beyond the first and last prototypes describes more extreme appearances. With this method, a change in feature slider value moves the veridical feature along its path between the prototypes altering size in situ. In comparison with Figure 3, changes to the shape of a thigh feature are defined by the path. Because the starting position for each veridical feature is defined with respect to its best fit-and, therefore, its proximity to any one of the prototypes - shape changes are described at a global level. If combined with the single-prototype method, the chosen appearance of different body parts can be interpreted in terms of body category. 
dimensional mesh (Boender, 1994; Sloan, 1993). This subdivides the area into a mosaic of triangular patches that can be treated in a uniform manner by the rendering algorithm. Bilinear interpolation maps each source pixel-red-green-blue bit planes of the color imageinto the corresponding location in the shape-changed image.

Deformation is smoothed by interpolating over adjoining body part boundaries by a few pixels, to avoid accidental discontinuities between body parts. The result of performing this operation over the entire image is shown in Figure 5. Shape change, tessellation, warping, and display are computed every time a slider event is generated.

\section{OPERATIONAL PARAMETERS}

\section{Options}

Run-time options can be supplied at the command line when executing the software. These influence the way in which images are presented and the kinds of graphical controls presented to the user.

Facial mask. As is shown in Figure 5, body figures can be displayed anonymously by overlaying a random circular mask on the head. The mask can be removed for debriefing purposes, as well as to demonstrate differences between anonymous and known body perception.

Selection of body parts. A list of body parts and their text labels determines which features can be manipulated on the interface. Body parts can be grouped to- gether (e.g., upper arm and forearm), and given more general names.

\section{Off-Line Operation}

The system may be used off line, as well as being an on-line experimental tool. This serves several important purposes. The experimenter is able to re-input observers' data, to recreate the chosen veridicality judgments and to take snapshots of the graphical window. During debriefing and demonstration, this has been a useful method of explaining perceptual choices to observers. Certain keystrokes in this mode permit the experimenter to toggle image display on or off, to superimpose the veridical and distorted body outlines in different colors and to reset body shape and sliders to veridical appearance. Presentation of interactive outline figures mimics the approach of Dickson-Parnell et al. (1987). The application displays body images by default but can be switched to outline mode during experiments.

\section{Control Stimuli}

To assess observers' performance using real body images, we obtain control data to measure consistency of behavior and response bias (Emery, Benson, CohenTovée, \& Tovée, 1995a, 1995b). Dressmakers' dummies were dressed in the leotards, and images were stored in a separate database. A child's dummy and a female dummy were used. Torso and hips were modified, using the internal mechanism, to provide impressions of ema-
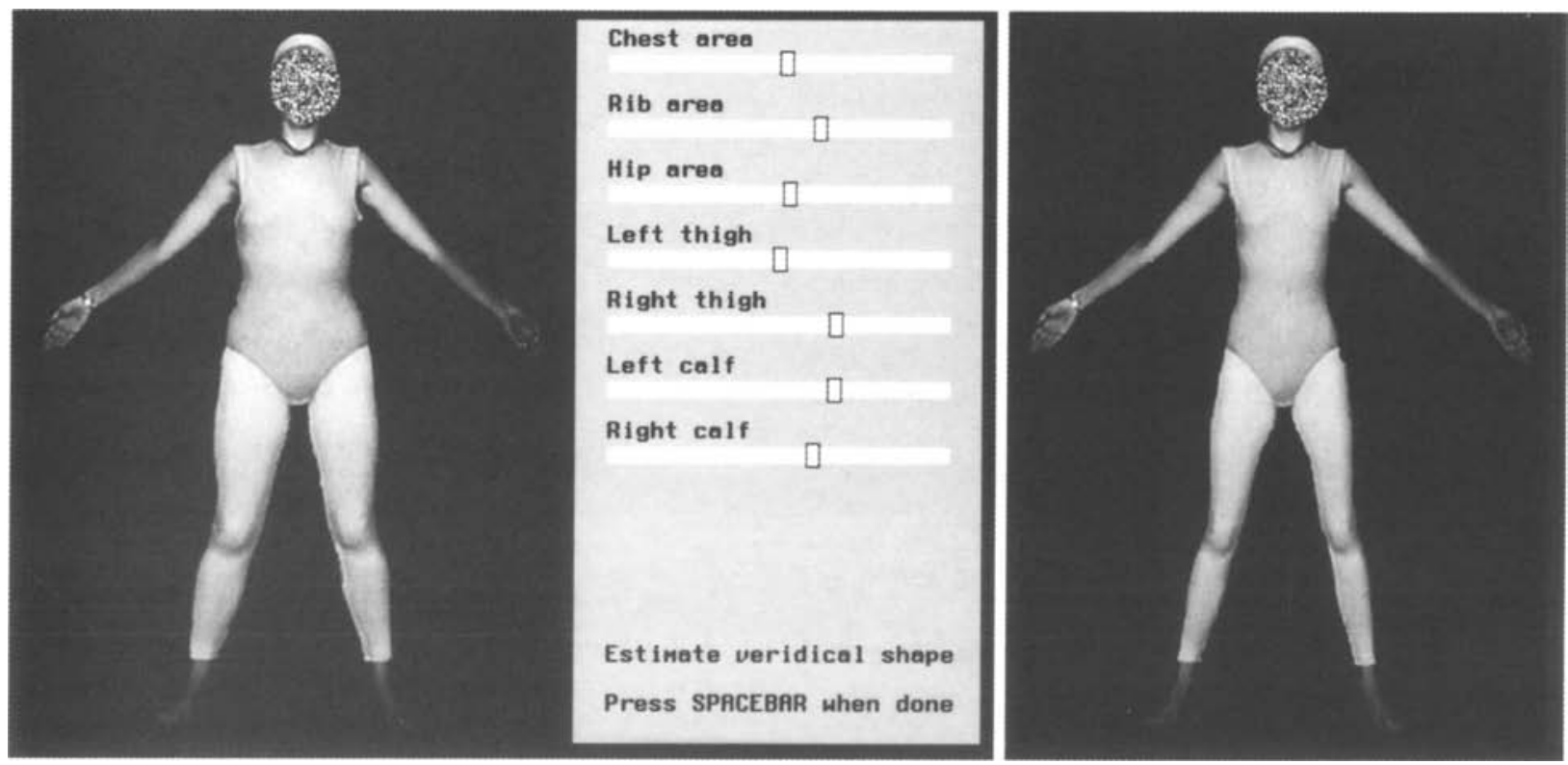

Figure 5. Shape changes using the graphical interface: A female figure is displayed wearing standard clothing. Facial identity can be masked during experiments. The left panel shows overestimations of veridical shape, using the single-prototype method. The right panel shows an underestimated figure shape. Observers are requested to estimate their veridical body shape by adjusting the sliders. Figures are presented at start-up, with each body part randomly offset from zero. Graphical sliders have asymmetric scales so that the zero-point is randomly offset to the left or the right. The goodness of veridical estimation is calculated in various ways and can be described in terms of both body parts and body type category (see the text). Changes made to individual body parts are made in an intuitive manner. Modern image-rendering techniques ensure that images of body shapes and surface textures will appear as natural as possible. 
ciation, normalcy, and obesity. Sliding bars inside the dummies allow the chest, waist, and hips to be altered. While the dummies are in view, observers adjust front and side control images until they settle on a perceived veridical match. This provides an index of visual acuity as well as of size-matching skills. Acuity can suffer as a result of chronic emaciation (Gilbert, Weiss, Sattler, \& Koch, 1990; Vandereycken, 1986; Yellowlees, Roe, Walker, \& Ben-Tovim, 1988).

Symmetry or deviations from symmetry and norms are also assessed with this technique. It is known that physical attractiveness and somatotype terms interact to influence judgments of body image (Norris, 1984; Tucker, 1984). Normal, mirror-reversed, left-left and right-right combinations of the observer's body are presented, to test whether perception of body image is motivated by particular body parts.

The first presentation of a body image can influence the observer (Ben-Tovim, Whitehead, \& Crisp, 1979). The effect of this starting point can be determined, and bias toward particular desired or undesirable somatotype images can be quantified. Placing the observer's own body parts in a random configuration at the start of a trial will indicate whether observer estimation follows patterns of distortion specific to particular body areas. This control method is an intrinsic part of our assessment procedure.

\section{Veridicality Index}

By providing a realistic image-based system, we believe we have overcome many of the limitations that have dogged previous approaches. From the outset, an important consideration was that the interface should be as intuitive as possible and changes in body shape should be believable. A naturalistic appearance achieves another important goal, which is to engage the observer as fully as possible and, thereby, design experiments that permit analysis of their self-perception of body shape (cf. Gardner, Morrell, Watson, \& Sandoval, 1990).

In off-line mode, we tabulate body part measures and slider values. Descriptive statistics show observer accuracy and correlation with prototypical body types and body parts. Automated systems such as this one and those reviewed in the introduction provide many ways to assess body image numerically. However, because each technique is very different - and some are very inaccurate and inflexible-it is difficult to find a correspondence between the measures that is independent of method.

Deviations from veridical are interpreted in two ways. First, estimation scores from graphical sliders appear as positive or negative values. As percentage deviations from zero, these are not indices of actual body mass over- or underestimation and so require some kind of translation.

Research in body size estimation has generally reported deviations from accuracy as percentages relative to actual body size (the Body Perception Index; Slade \& Russell, 1973). A 20\% overestimation score would imply that the subject perceived his or her body as being $20 \%$ larger than it actually was. Ben-Tovim and colleagues (Ben-Tovim et al., 1979) point out that this formula-perceived size divided by real size-results in artificially high under- or overestimation scores for small-bodied subjects (e.g., anorexics), owing to the small denominator term. Instead, it is preferable to report deviations from accuracy in more absolute values. It is important that we know whether an observer underestimates or overestimates his or her size and whether he or she does so to a lesser or a greater degree than the next person. The utility of percentage scores may be limited. Percentage scores are rather general and need to be treated with some caution. At the very least, scores have to be averaged across different body views and may be very different for each body part.

An alternative measure involves expression of veridicality in real-world units. The calibration bar information is used to express $\mathrm{cm}^{2}$ deviations of body part area in front and side views. Having access to such information provides a more accurate indication of over- and underestimation of size (Figure 6). Knowing the size of features in the veridical and distorted images means that we can consider body size at local and global levels. Output area differences are accompanied by slider values. Correlations between veridical and distorted body parts and body wholes provide a goodness-of-fit statistic $\left(R^{2}\right)$, and those body parts deviating significantly from veridical and prototype(s) are identified.

This strategy is preferred, because we can relate area measures to physical measurements of the body. Metric (cm) and imperial (in.) measurements are used when assessing body shape (recall that BMI units are in $\mathrm{kg} / \mathrm{m}^{2}$ ) and size and fit of clothing. Self-perception of body image is frequently motivated by measurement. This

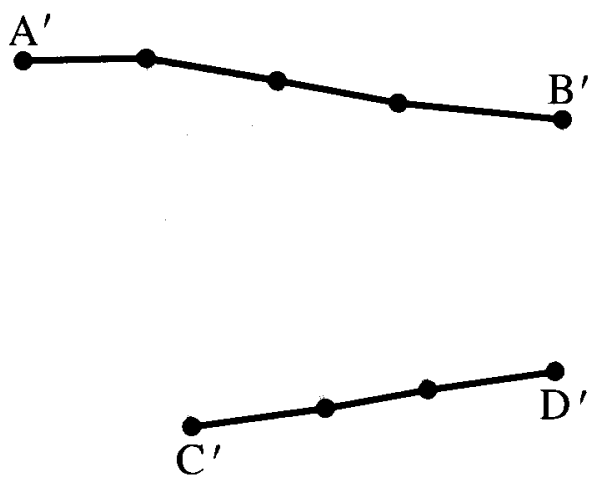

Figure 6. Body part area: Judgments of body size are quantified, using several indices of veridicality. Over- and underestimations of appearance are quantified by slider values (percentage deviations from true shape) and calculations of feature area in the image plane. Since the image-rendering subsystem performs texture mapping by triangulating each body part in turn, the mosaics are also used to approximate surface area. Calibration markers in the room (outside the cropped image area in Figure 5) are used to calculate values in pixels ${ }^{2}$, and the result is converted to $\mathrm{cm}^{2}$. Real-worid measures are useful descriptions, because body and clothing sizes are also measured in these units. 
seems a sensible viewpoint, because we are able to empower measurement in terms of perceived body parts of veridical bodies and prototypes.

\section{Classification of Body Prototypes}

As we suggested earlier, it has been possible to refine definition of the discrete BMI categories. The basis for this was the measurement of body part areas from each view of males and females in the database. For simplicity, a stand-alone software application can be built, using conditional compilation of the source code. This provides a stripped-down program that uses the same internal routines as the body image software but does not use graphical routines.

We measured the areas of body parts from the front and the side + arms (arms up) views. Males and females were analyzed separately. Measures were analyzed with Bayesian statistics (Box \& Tiao, 1973). Such an approach is used to discover structure in large-scale data sets (Cheeseman \& Stutz, 1996; Wallace \& Boulton, 1969; Wallace \& Freeman, 1987). Bodies categorized in this way yielded classes of body shape that contain self-similar shapes. Class averages are then used as prototypes for the multiple-prototypes model. The advantage of this approach has been that we can understand how the appearance of each body part influences differences between categories of body type.

This is a significant advance over other methods of classification and avoids abrupt changes between body categories that will affect the veracity of changes in body shape using the graphical interface. Veridicality judgments can now be expressed in terms of statistical categories of body type.

\section{IMPLEMENTATION CONSIDERATIONS}

Graphical sliders are not presented with gradations or numerical read-out. Additional command line options allow the sign of the slider to be swapped randomly -for example, $-50 \%$ to $+50 \%$ on one slider, and $+50 \%$ to $-50 \%$ on another, and so on. Asymmetric ranges can be specified within particular bounds (or assigned randomly) - for example, $-60 \%$ to $+45 \%$. We do this so that observers cannot default to the center of the slider, assuming that it represents true veridical shape, and thereby falsely obtain estimation scores close to $100 \%$ correct. In any case, this is not the individual's task. This is of considerable assistance in addressing problems having to do with choice of the starting point in such experiments (Ben-Tovim et al., 1979).

The simplest course that an experiment might take is merely for the observer to make estimates of their veridical body shape. Only a small number of adjustments are required to complete a trial. Randomizing slider starting positions is a reasonable way to make repeated measures of an individual's selections, and using single and multi- ple prototypes, mirror-reflected body views, and control images adds considerably to the reliability of the data.

We have yet to observe difficulty with usage of the graphical interface. Sliders seem an appropriate way for observers to manipulate their body image as a system of related parts. Less than perfect reliability may reflect the possibility that body image may not be stable over time but may be affected by physiological, emotional, and situational factors, such as depressed mood (Cohen-Tovée, 1993; Cooper \& Taylor, 1988), time of day, hunger, motivation, experimenter sex and familiarity (Meerman, Vandereycken, \& Napierski, 1986) and room lighting conditions (Button, Fransella, \& Slade, 1977). Some of these factors are difficult to control precisely. Slade (1985) has proposed that body part methods tap a more stable component of body image than do holistic techniques and may, therefore, demonstrate higher reliability. Our approach integrates these issues.

The choice of system platform was dictated by the availability of high-performance CPU and graphics support at the time. A consortium of industry leaders now provides OpenGL graphics libraries and video cards that have built-in hardware support. It should be noted that most of the software is numerical and only the interface requires direct graphical support. However, the availability of high-performance PCs and 32-bit operating systems such as Linux, which has full device and library support for OpenGL, persuades us to continue developing our software in this environment. Linux also supports X-windows and SVGA graphics libraries, all of which are freely available.

\section{DISCUSSION}

The system we have described improves on previous techniques. Realistic simulation of weight gain or loss is achieved in a procedure that allows independent, interactive manipulation of separate body parts while providing holistic feedback of body image. Feedback in the form of a recognizable image of the subject may have the added advantage of making the task less difficult than one in which body widths have to be imagined. Other parts methods have relied entirely on observers' mental representation of their bodies and depend largely on recall memory. Where an image is provided, subjects are further aided by recognition memory.

In practice, modeling and rendering of body image has been successful. Male and female observers have been studied. Our database currently comprises $120+$ male and $200+$ female bodies in at least front and side + arms views. The way in which deposition or absence of tissue in body parts has been modeled has been very useful (Emery et al., 1995a, 1995b; Maisey, Emery, Benson, Cohen-Tovée, \& Tovée, 1996). As an estimate of body size, the technique cannot be taken to measure every mental facet of body image (Hsu \& Sobkiewicz, 
1991). This has a clear effect on the issues of body image as a mental construct. Here, we address the perceptual aspect and claim only to measure this component.

The technique represents an innovation in the areas of body image and body size perception, using modern techniques in computer graphics. The procedure also offers a means of categorizing subjects into body groups that may be preferable to formulaic techniques such as Body Mass Index, which is based on only two parameters. We propose to use this method to establish the boundaries and content of somatotype categories, with the aim of generating fewer "wrong" categorizations. Combined with careful experimental design, the bodymorphing technique should prove an invaluable tool in future research into eating disorders and perception of body image.

\section{REFERENCES}

AsKevold, F. (1975). Measuring body image: Preliminary report of a new method. Psychotherapy \& Psychosomatics, 26, 71-77.

BENSON, P. J. (1992). Perception and recognition of computer-enhanced facial attributes and abstracted prototypes. Unpublished doctoral dissertation, University of St. Andrews.

BENSON, P. J. (1994a). Morph transformation of the facial image. Image \& Vision Computing, 12, 691-696.

BENSON, P. J. (1994b). On facial image composite prototyping as a descriptive and diagnostic tool. Journal of Audiovisual Media in Medicine, 17, 27-30.

Benson, P. J., \& Perrett, D. I. (1991a). Perception and recognition of photographic quality caricatures: Implications for the recognition of natural images. European Journal of Cognitive Psychology, 3, 105 135.

Benson, P. J., \& Perrett, D. I. (199lb). Synthesizing continuous-tone caricatures. Image \& Vision Computing, 9, 123-129.

Benson, P. J., \& Perret, D. I. (1993). Extracting prototypical facial images from exemplars. Perception, 22, 257-262.

Ben-Tovim, D. I., Whitehead, J., \& CRISP, A. H. (1979). A controlled study of the perception of body width in anorexia nervosa. Journal of Psychosomatic Research, 23, 267-272.

BOENDER, E. (1994). Reliable Delaunay-based mesh generation and mesh improvement. Communications in Numerical Methods in Engineering, 10, 773-783.

Box, G. E. P., \& TiaO, G. C. (1973). Bayesian inference in statistical analysis. Chichester, U.K.: Wiley.

Button, E. J., Fransella, F., \& Slade, P. D. (1977). A reappraisal of body perception disturbance in anorexia nervosa. Psychological Medicine, 7, 235-243.

Cheeseman, P., \& Stutz, J. (1996). Bayesian classification (AutoClass): Theory and results. In U. M. Fayyad, G. Piatetsky-Shapiro, P. Smyth, \& R. Uthurusamy (Eds.), Advances in knowledge discovery and data mining (pp. 61-83). Cambridge, MA: MIT Press, AAAI Press.

CoHEn-TovéE, E. M. (1993). Depressed mood and concern with weight and shape in normal young women. International Journal of Eating Disorders, 14, 223-227.

COOPER, P. J., \& TAYLOR, M. J. (1988). Body image disturbance in bulimia nervosa. British Journal of Psychiatry, 153, 20-22.

Dickson-Parnell, B., Jones, M., Braddy, D., \& Parnell, C. P. (1987). Assessment of body image perceptions using a computer program. Behavior Research Methods, Instruments, \& Computers, 19, 353-354.

Emery, J. L., Benson, P. J., Cohen-Tovée, E. M., \& Tovée, M. J. (1995a). A computerised measure of body image. Brain Research Association Abstracts, 12, 68

Emery, J. L., Benson, P. J., Cohen-Tovée, E. M., \& Tovée, M. J. (1995b). A computerised measure of body shape perception. $A p$ petite, 24, 279.
Freeman, R. J., Thomas, C. D., Solyom, L., \& Hunter, M. (1984). A modified video camera for measuring body image distortion: Technical description and reliability. Psychological Medicine, 14, 411416.

Gardner, R. M., Morrell, J. A., Watson, D. N., \& Sandoval, S. L. (1990). Eye movements and body size judgements in the obese. International Journal of Eating Disorders, 9, 537-544.

Gilbert, J. M., Weiss, J. S., Sattler, A. L., \& Кoch, J. M. (1990). Ocular manifestations and impression cytology of anorexia nervosa. Ophthalmology, 97, 1001-1007.

Glucksman, M. L., \& Hirsch, J. (1969). The response of obese patients to weight reduction. Psychosomatic Medicine, 31, 1-7.

Hsu, L. G., \& SobkiEwicz, T. A. (1991). Body image disturbance: Time to abandon the concept for eating disorders? International Journal of Eating Disorders, 10, 15-30.

JansSEN, B., \& Whiting, H. T. (1984). Sheldon's physical-psychical typology revisited. Journal of Research in Personality, 18, 432-441.

Kellet, J., Marzillier, J. S., \& Lambert, C. (1981). Social skills and somatotype. British Journal of Medical Psychology, 54, 149-155.

Maisey, D. S., Emery, J. L., Benson, P. J., Cohen-Tovée, E. M., \& TovéE, M. J. (1996, March). Assessment of male body shape satisfaction using computerised caricaturing techniques. Paper presented at the annual meeting of the Brain Research Association, Newcastle, U.K.

Meerman, R., Vandereycken, W., \& Napierski, C. (1986). Methodological problems of body image research in anorexia nervosa patients. Acta Psychiatrica Belgica, 86, 42-51.

NoRRIS, D. L. (1984). The effects of mirror confrontation on selfestimation of body dimensions in anorexia nervosa, bulimia and two control groups. Psychological Medicine, 7, 253-257.

Reitman, E. E., \& Cleveland, S. E. (1964). Changes in body image following sensory deprivation in schizophrenic control groups. Journal of Abnormal \& Social Psychology, 68, 168-176.

Schlundt, D. G., \& Bell, C. (1993). Body Image Testing System: A microcomputer program for assessing body image. Journal of Psychopathology \& Behavioural Assessment, 15, 267-285.

SLADE, P. D. (1985). A review of body image studies in anorexia nervosa and bulimia nervosa. Journal of Psychiatric Research, 19, 255-265.

Slade, P. D., \& Russell, G. F. M. (1973). Awareness of body dimensions in anorexia nervosa: Cross-sectional and longitudinal studies. Psychological Medicine, 3, 188-199.

SLOAN, S. W. (1993). A fast algorithm for generating constrained Delaunay triangulations. Computers \& Structures, 47, 441-450.

TAYlor, M., Baldwin, J., \& CoOPER, P. (1985). Measurement of body image in anorexia nervosa. British Journal of Psychiatry (Correspondence), 146, 214-215.

Thompson, J. K., \& Spana, R. E. (1988). The adjustable light beam method for the assessment of size estimation accuracy: Description, psychometric and normative data. International Journal of Eating Disorders, 7, 521-526.

Traub, A. C., \& Orbach, J. (1964). Psychophysical studies of body image: I. The adjustable body distorting mirror. Archives of General Psychiatry, 11, 53-66.

TUCKer, L. A. (1984). Physical attractiveness, somatotype and the male personality: A dynamic interactional perspective. Journal of Clinical Psychology, 40, 1226-1234.

VANDEREYCKEN, W. (1986). Anorexia nervosa and visual impairment. Comparative Psychiatry, 27, 545-548.

Wallace, C. S., \& Boulton, D. M. (1969). An information measure for classification. Computer Journal, 11, 185-194.

WALlace, C. S., \& FreEMAN, P. R. (1987). Estimation and inference by compact encoding. Journal of the Royal Statistical Society B, 49, 240-265.

Wolberg, G. (1990). Digital image warping. Los Alamitos, CA: IEEE Computer Society Press.

Yellowlees, P. M., Roe, M., WAlker, M. K., \& BeN-Tovim, D. I. (1988). Abnormal perception of food size in anorexia nervosa. British Medical Journal, 296, 1689-1690.

(Manuscript received May 1, 1998; revision accepted for publication January $7,1999$. .) 